\title{
Effects of spatial resolution of remotely sensed data on estimating urban impervious surfaces
}

\author{
Weifeng $\mathrm{Li}^{1}$, Zhiyun Ouyang ${ }^{1, *}$, Weiqi Zhou ${ }^{2}$, Qiuwen Chen ${ }^{1}$ \\ 1. State Key Laboratory of Urban and Region Ecology, Research Centre for Eco-Environmental Sciences, Chinese Academy of Sciences, Beijing \\ 100085, China. E-mail: rceelwf@yahoo.com.cn \\ 2. Department of Plant Sciences, University of California, Davis, Mail Stop 1, 1210 PES, One Shields Ave., Davis, CA 95616, USA
}

Received 20 September 2010; revised 13 December 2010; accepted 20 December 2010

\begin{abstract}
Impervious surfaces are the result of urbanization that can be explicitly quantified, managed and controlled at each stage of land development. It is a very useful environmental indicator that can be used to measure the impacts of urbanization on surface runoff, water quality, air quality, biodiversity and microclimate. Therefore, accurate estimation of impervious surfaces is critical for urban environmental monitoring, land management, decision-making and urban planning. Many approaches have been developed to estimate surface imperviousness, using remotely sensed data with various spatial resolutions. However, few studies, have investigated the effects of spatial resolution on estimating surface imperviousness. We compare medium-resolution Landsat data with high-resolution SPOT images to quantify the imperviousness in Beijing, China. The results indicated that the overall $91 \%$ accuracy of estimates of imperviousness based on TM data was considerably higher than the $81 \%$ accuracy of the SPOT data. The higher resolution SPOT data did not always predict the imperviousness of the land better than the TM data. At the whole city level, the TM data better predicts the percentage cover of impervious surfaces. At the sub-city level, however, the ring belts from the central core to the urban-rural peripheral, the SPOT data may better predict the imperviousness. These results highlighted the need to combine multiple resolution data to quantify the percentage of imperviousness, as higher resolution data do not necessarily lead to more accurate estimates. The methodology and results in this study can be utilized to identify the most suitable remote sensing data to quickly and efficiently extract the pattern of the impervious land, which could provide the base for further study on many related urban environmental problems.
\end{abstract}

Key words: remote sensing; impervious surface; landscape pattern; spatial resolution; object-based image analysis; urban landscape DOI: $10.1016 / \mathrm{S} 1001-0742(10) 60541-4$

Citation: Li W F, Ouyang Z Y, Zhou W Q, Chen Q W, 2011. Effects of spatial resolution of remotely sensed data on estimating urban impervious surfaces. Journal of Environmental Sciences, 23(8): 1375-1383

\section{Introduction}

Impervious surfaces are those that do not allow water to infiltrate to the soil. Impervious surfaces are primarily associated with human transportation (streets, highways, parking lots, and sidewalks) and rooftops of buildings, which represent the imprint of land development on the landscape. Therefore, imperviousness is generally used to quantify and map the degree of urbanization and the extent of urban land use (Civco et al., 2002; Yang et al., 2003; Bauer et al., 2004). More importantly, imperviousness is a useful environmental indicator that can be used to measure the impacts of urbanization on surface runoff, water quality, air quality, biodiversity and microclimate (White and Greer, 2006; Luo et al., 2009; Lee et al., 2010). In addition, imperviousness is one of the few variables that can be explicitly quantified, managed and controlled at each stage of land development. Therefore, accurate

\footnotetext{
* Corresponding author. E-mail: zyouyang@rcees.ac.cn
}

estimation of impervious surfaces is critical for urban land management decision-making, ecosystem monitoring and urban planning.

Ground surveys and field measurements are traditionally used to estimate surface imperviousness and to subsequently generate maps of impervious surfaces. These approaches, however, are often very labor intensive, and thus expensive and difficult to implement for large areas. Remote sensing has long been used for land cover/use classification. It allows for up-to-date, spatially explicit estimates of urban surface imperviousness over large areas.

Many methods have been developed to measure urban imperviousness using ground measurements and remotely sensed data including multiple regression analysis (Ridd, 1995; Xiao et al., 2007), spectral unmixing (Ward et al., 2001; Philip and Roberts, 2003; Lu and Weng, 2006), artificial neural networks (Wang et al., 2000; Flanagan and Civco, 2001), and classification trees (Goetz et al., 2001). Xiao et al. (2007) used regression tree model to estimate impervious surface using Landsat images, and 
found that normalized band ratios best predicted the intensity of urbanization. Flanagan and Civco (2001) applied an artificial neural network method to map sub-pixel imperviousness using an ETM+ image. Wu (2004) developed a normalized spectral mixture analysis (SMA) method based on an ETM+ image to examine the percentage of urban impervious surfaces.

These approaches have proved to be valuable in monitoring and estimating surface imperviousness. However, applications of these approaches, particularly the SMA methods, remain challenging in heterogeneous urban areas. SMA methods require identification of spectrally pure pixels, known as endmembers. In highly heterogeneous urban settings, there are considerable spectral variations in impervious surfaces, ranging from low to high albedo. Consequently, it is very difficult to identify pure endmembers. This is particularly true for remote sensing data at low to medium resolutions.

In the present study, a practical method was developed to assess the pattern of urban impervious surfaces from remotely sensed data. We compared medium-resolution TM data with high-resolution SPOT images to quantify the imperviousness in Beijing, China. The objectives of this study were: (1) to develop an appropriate model to estimate imperviousness using different types of remote sensing data; (2) to quantify the pattern of urban impervious surfaces derived from multi-resolution remote sensing data; and (3) to analyze the effects of spatial resolution of remote sensing data on the impervious surface pattern.

\section{Data and methods}

\subsection{Study area}

This research focused on the densely urbanized inner city of Beijing, China (Fig. 1). The study area was about $670 \mathrm{~km}^{2}$. The inner city of Beijing has an average elevation of less than $50 \mathrm{~m}$, and has a temperate, humid, monsoon continental climate. The expansion of urban areas in Beijing followed a typical concentric mode. It forms

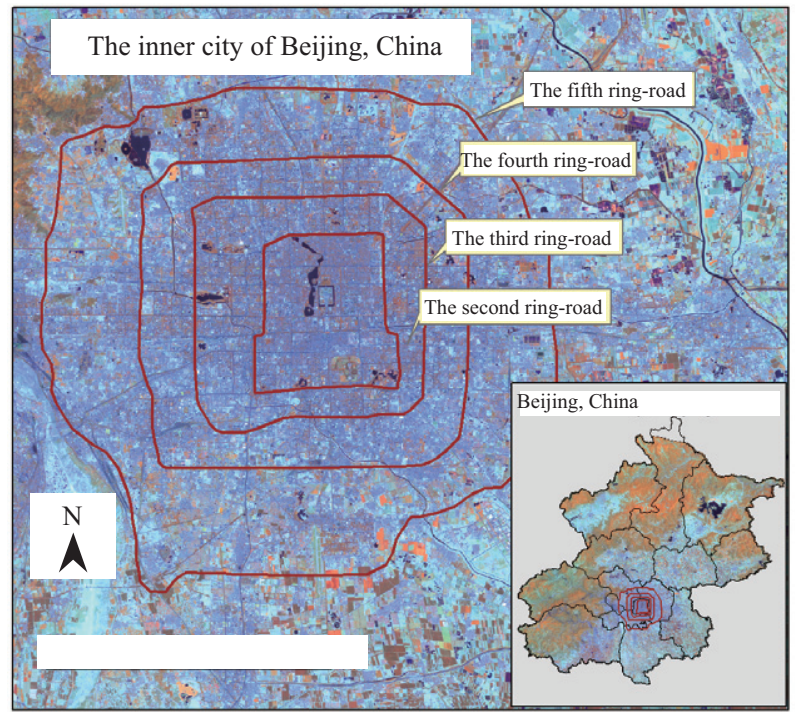

Fig. 1 Study site. concentric circles from the inner centre (the second ring road) to the outskirts (the fifth ring highway). Different ring belts represent different development patterns. The oldest part of Beijing has a history going back more than 3000 years and lies within the second ring road. It has many historic sites and old residential communities. Land between the third and fourth ring roads was developed in recent decades, and contains various land uses, including residential areas, commercial and business centres, universities, and government and institutional buildings. The urban-rural transition zones, which are experiencing rapid urbanization, lie between the fourth and fifth ring roads. Due to their different development processes, the patterns of land cover/use differ greatly between these ring belts. Consequently, their patterns of impervious surfaces differ considerably.

\subsection{Data}

This study used Landsat TM imagery with $30 \mathrm{~m}$ resolution, and SPOT imagery with $10 \mathrm{~m}$ resolution to estimate surface imperviousness. Both datasets were acquired in May 2004. In addition, Quickbird imagery with a spatial resolution of $0.61 \mathrm{~m}$, was used as reference data for model calibration and validation. The image data were also collected in May 2004.

\subsection{Statistical analysis}

\subsubsection{Sampling strategy}

Medium-resolution TM images, high-resolution SPOT images and samples of high-resolution images (Quickbird images, $0.61 \mathrm{~m}$ resolution) were utilized. Training data were sampled with a stratified random sampling methodology, while validation data were sampled in a purely random fashion. The sampling procedure was performed for each individual percentage surface target dataset after areas of cloud, cloud shadow, and haze were masked out and replaced with useful spectral information.

From the land use/cover characteristics of the study site, 200 object units (total area $56 \mathrm{~km}^{2}$ ) based on objectoriented analysis of remote sensing images were randomly selected as the samples for principal component regression (PCR) analysis to develop the imperviousness estimation model. Compared with the pixel-based classification method, the object-oriented classification approach could provide another means by which to integrate spatial information. Spatial information such as texture, shape, and context can be added to increase the discrimination between spectrally similar types of land cover. Using this approach, the remote sensing image was first segmented into small objects such as building blocks or road segments according to their shape, texture, context and spatial relations as well as spectral feature (Zhou et al., 2008; Zhou and Troy, 2008). In this study, object-oriented segmentation was carried out to classify pixels into basic "objects", which are the fundamental units for subsequent estimation of the surface imperviousness based on the imperviousness index. 


\subsubsection{Indicator selection}

In this study, we selected six indices: the normalized difference of vegetation index (NDVI), normalized built-up index (NDBI), average brightness, maximum difference of brightness, standard deviation of brightness, and the ratio of brightness based on previous publications (Yuan and Bauer, 2007; Zhou and Troy, 2008).

NDVI is one of the most widely used vegetation indices for assessment and monitoring of diverse vegetation cover (Fernandez, 2010). It is defined as Eq. (1):

$\mathrm{NDVI}=\frac{\mathrm{NIR}-\mathrm{RED}}{\mathrm{NIR}+\mathrm{RED}}$

where, RED is the reflectance in the red waveband, and NIR is the reflectance in the near-infrared waveband.

The NDBI index was proposed by Zha et al. (2003), and can be used to effectively map built-up areas. This index is defined as Eq. (2):

$\mathrm{NDBI}=\frac{\mathrm{SWIR}-\mathrm{NIR}}{\mathrm{SWIR}+\mathrm{NIR}}$

where, SWIR is the reflectance in the shortwave infrared waveband, and NIR is the reflectance in the near-infrared waveband.

Brightness $(B)$ is calculated as the channel mean value of multiple spectral bands (Eq. (3)):

$B=\frac{1}{n_{\mathrm{L}}} \times \sum_{i=1}^{n_{\mathrm{L}}} \overline{c_{i}}$

where, $n_{\mathrm{L}}$ is the number of spectral bands, and $\overline{c_{i}}$ is the mean value band $i$.

The maximum difference of brightness (max diff.) index is the difference between the minimum mean value of an object and its maximum value. It is normalized by the brightness.

The standard deviation of brightness is a measure of how far apart the data are from the average of the data. It is calculated by Eq. (4):

$\sigma_{\mathrm{L}}=\sqrt{\frac{1}{n-1} \sum\left(c_{\mathrm{L} i}-\overline{c_{\mathrm{L}}}\right)^{2}}$

where, $\sigma_{\mathrm{L}}$ is the standard derivation of brightness, $n$ represents the number of pixel cells within the target unit, $c_{\mathrm{L} i}$ represents the digital value of cell $i$ from band $\mathrm{L}$, and $\overline{c_{\mathrm{L}}}$ represents the average digital value of band $\mathrm{L}$ for all target pixel cells.

The ratio of brightness $\left(r_{\mathrm{L}}\right)$ is the mean value of a certain band for an image object divided by the sum of the mean values of all the spectral bands. It is calculated as Eq. (5):

$r_{\mathrm{L}}=\frac{\overline{C_{\mathrm{L}, \text { Object }}}}{\overline{C_{\mathrm{L}, \mathrm{SO}}}}$

where, $C_{\mathrm{L}, \text { Object }}$ represents the average brightness of band $\mathrm{L}$ for the target object unit, and $\overline{C_{\mathrm{L}, \mathrm{SO}}}$ represents the average brightness of all the selected bands for the target object unit.

In total, 22 indictors derived from the TM image were selected. The relativity analysis indicated that all 22 multispectral indices have either relatively strong or weak relationships with the surface imperviousness (Table 1).

\subsubsection{Correlation analysis and principal component analysis}

Multiple linear regression (MLR) analysis is one of the most widely used methodologies for expressing the dependence of a response variable on independent variables. Despite its success in numerous applications, the regression approach faces difficulties when the independent variables are correlated with each other (Abdul-Wahab et al., 2005). Multi-collinearity, or high correlation among the independent variables in a regression equation, can make it difficult to correctly identify the most important contributions to a physical process. One method of removing such multi-collinearity is to use multi-variate data analysis (MDA). One specific type of MDA method is principal component analysis (PCA), which involves a mathematical procedure that transform the possibly correlated variables into statistically independent variables called principal components, and related to the original variables by an orthogonal transformation (Statheropoulos et al., 1998; Vaidya et al., 2000). This transformation is defined in such a way that the first principal component has highest variance (that is, accounts for as much of the variability in the data as possible), and each succeeding component in turn has the highest variance under the constraint that would be orthogonal to the preceding components. PCA is equally useful in regression analysis for mitigating the problem of multicollinearity and in exploring the relationships among the independent variables. The new uncorrelated variables from the PCA become ideal to use as predictors in a principal component regression (PCR). $\mathrm{PCR}$ is a regression analysis that uses principal component analysis when estimating regression coefficients. It is a procedure used to overcome problems which arise when the exploratory variables are close to being collinear.

Thus, considering the relationships between the multispectral indicators from images, in the present study, PCR analysis was used to explore the relationships between surface imperviousness and multi-spectral indicators and thus to develop models for estimation of surface imperviousness. The accuracy of the model was assessed by comparison with the true land imperviousness of the sample units that were tested. The standard error analysis was conducted to evaluate its reliability. Finally, the spatial patterns of the urban impervious surfaces were extracted by applying this model.

The main steps in developing the model to estimate surface imperviousness included: (1) The image pixels are first segmented into objects which are the basic units for estimating land imperviousness by applying the eCognition software which embedded image segmentation algorithms. (2) Sampling land units are created using the stratified random sampling methodology as the training data and testing data. (3) The multi-spectral indicators of all the sampling units are calculated using the eCognition platform. (4) The relationships between the multi-spectral indicators and land imperviousness are explored. The PCR analysis is carried out to eliminate the significant relationships between the multi-spectral indicators by applying SPSS software. The models to estimate the imperviousness 
Table 1 Relationships between impervious index and TM multi-spectral factors

\begin{tabular}{|c|c|c|c|c|c|c|c|c|c|c|}
\hline & $\ln (b 1)$ & $\ln (b 2)$ & $\ln (b 3)$ & $\ln (b 4)$ & $\ln (b 5)$ & $\ln (b 6)$ & Ratio1 & Ratio2 & Ratio3 & Ratio4 \\
\hline Imp VIOUS & $0.444 * *$ & $0.315^{* *}$ & $0.348^{* *}$ & $-0.618 * *$ & -0.149 & $0.187 *$ & $0.448^{* *}$ & $0.508^{* *}$ & $0.693^{* *}$ & $-0.742 * *$ \\
\hline $\ln (b 1)$ & & $0.976^{* *}$ & $0.968^{* *}$ & 0.149 & $0.725^{* *}$ & $0.895^{* *}$ & $-0.303 * *$ & $0.240 * *$ & $0.779^{* *}$ & $-0.694 * *$ \\
\hline $\ln (b 2)$ & & & $0.991 * *$ & $0.303^{* *}$ & $0.834 * *$ & $0.950^{* *}$ & $-0.471 * *$ & 0.131 & $0.719^{* *}$ & $-0.592 * *$ \\
\hline $\ln (b 3)$ & & & & $0.240^{* *}$ & $0.813^{* *}$ & $0.958^{* *}$ & $-0.460 * *$ & 0.135 & $0.764^{* *}$ & $-0.647 * *$ \\
\hline $\ln (b 4)$ & & & & & $0.725^{* *}$ & $0.407^{* * *}$ & $-0.811 * *$ & $-0.556^{* *}$ & $-0.333^{* *}$ & $0.543^{* *}$ \\
\hline $\ln (b 5)$ & & & & & & $0.916^{* *}$ & $-0.829 * *$ & $-0.291 * *$ & $0.302 * *$ & -0.145 \\
\hline $\ln (b 6)$ & & & & & & & $-0.639 * *$ & -0.084 & $0.603^{* *}$ & $-0.503^{* *}$ \\
\hline Ratio1 & & & & & & & & $0.533^{* *}$ & 0.056 & $-0.211^{* *}$ \\
\hline \multirow{4}{*}{$\begin{array}{l}\text { Ratio2 } \\
\text { Ratio3 } \\
\text { Ratio4 }\end{array}$} & & & & & & & & & $0.463^{* *}$ & $-0.499 * *$ \\
\hline & & & & & & & & & & $-0.864 * *$ \\
\hline & & & & & & & & & & \\
\hline & Ratio5 & Ratio6 & std 1 & std2 & std3 & std4 & std5 & std6 & NDBI & NDVI \\
\hline Imp VIOUS & $0.727 * *$ & $0.250^{* *}$ & $-0.332 * *$ & $-0.336^{* *}$ & $0.406^{* *}$ & $0.533^{* *}$ & $-0.340 * *$ & -0.380 ** & $0.560^{* *}$ & $-0.746 * *$ \\
\hline $\ln (b 1)$ & -0.141 & $0.676^{* *}$ & 0.048 & 0.057 & -0.030 & $-0.184 *$ & 0.040 & -0.054 & $0.840^{* *}$ & $-0.770^{* *}$ \\
\hline $\ln (b 2)$ & 0.009 & $0.705^{* *}$ & 0.064 & 0.098 & 0.036 & -0.111 & 0.109 & 0.011 & $0.803^{* *}$ & $-0.683^{* *}$ \\
\hline $\ln (b 3)$ & -0.020 & $0.750^{* * *}$ & 0.027 & 0.074 & 0.025 & -0.154 & 0.115 & 0.024 & $0.851^{* *}$ & -0.736 ** \\
\hline $\ln (b 4)$ & $0.776^{* *}$ & 0.144 & $0.465^{* *}$ & $0.461 * *$ & $0.489 * *$ & $0.526^{* *}$ & $0.379 * *$ & $0.404 * *$ & $-0.223 * *$ & $0.481^{* *}$ \\
\hline $\ln (b 5)$ & $0.512 * *$ & $0.668^{* *}$ & $0.288^{* *}$ & $0.319^{* *}$ & $0.307 * *$ & $0.213^{* *}$ & $0.342 * *$ & $0.287 * *$ & $0.510^{* *}$ & $-0.228 * *$ \\
\hline $\ln (b 6)$ & $0.204 * *$ & $0.826^{* *}$ & 0.137 & $0.183^{*}$ & 0.155 & -0.040 & $0.230^{* *}$ & $0.171^{*}$ & $0.789^{* *}$ & $-0.581 * *$ \\
\hline Ratio1 & $-0.712 * *$ & $-0.468 * *$ & $-0.274 * *$ & $-0.333 * *$ & $-0.396^{* *}$ & $-0.343^{* *}$ & $-0.386^{* *}$ & $-0.388^{* *}$ & $-0.160 *$ & -0.150 \\
\hline Ratio2 & $-0.602 * *$ & -0.122 & $-0.416^{* *}$ & $-0.437 * *$ & $-0.516^{* *}$ & $-0.447 * *$ & $-0.403^{* *}$ & $-0.524 * *$ & $0.282^{* *}$ & $-0.508^{* *}$ \\
\hline Ratio3 & $-0.507 * *$ & $0.527^{* *}$ & $-0.277 * *$ & $-0.222 * *$ & $-0.272 * *$ & $-0.441 * *$ & -0.137 & $-0.244 * *$ & $0.842^{* *}$ & $-0.922 * *$ \\
\hline Ratio4 & $0.578 * *$ & $-0.541^{* *}$ & $0.285^{* *}$ & $0.260 * *$ & $0.329 * *$ & $0.519 * *$ & $0.181 *$ & $0.271 * *$ & $-0.884 * *$ & $0.965^{* *}$ \\
\hline Ratio5 & & 0.127 & $0.372^{* *}$ & $0.392 * *$ & $0.451^{* *}$ & $0.568^{* *}$ & $0.465^{* *}$ & $0.468 * *$ & $-0.245^{* *}$ & $0.560^{* *}$ \\
\hline Ratio6 & & & 0.103 & 0.155 & 0.151 & -0.122 & $0.240 * *$ & $0.231^{* *}$ & $0.767 * *$ & -0.580 *** \\
\hline Std1 & & & & $0.962^{* *}$ & $0.900^{* *}$ & $0.594^{* *}$ & $0.686^{* *}$ & $0.751^{* *}$ & $-0.170^{*}$ & $0.295^{* *}$ \\
\hline Std2 & & & & & $0.971 * *$ & $0.624 * *$ & $0.789 * *$ & $0.845^{* *}$ & -0.123 & $0.251^{* *}$ \\
\hline Std3 & & & & & & $0.667^{* *}$ & $0.834^{* *}$ & $0.915^{* *}$ & $-0.174 *$ & $0.315^{* *}$ \\
\hline Std4 & & & & & & & $0.741 * *$ & $0.664 * *$ & $-0.357 * *$ & $0.509^{* *}$ \\
\hline Std5 & & & & & & & & $0.927^{* *}$ & 0.011 & $0.159^{*}$ \\
\hline Std6 & & & & & & & & & -0.098 & $0.258^{* *}$ \\
\hline NDBI & & & & & & & & & & $-0.924 * *$ \\
\hline
\end{tabular}

Imp: percentage of impervious surface; $\ln (\mathrm{b} 1)-\ln (\mathrm{b} 6)$ : 1-6 spectral channels of TM image by standardization; Ratio1-Ratio6: ratio of brightness of 1-6 spectral channels of TM image; std1-std6: standard derivation of brightness of channels 1-6 of TM image.

of the land are then developed by combining the different independent spectral indicators. Finally, the model with the best regression effect is selected to estimate the imperviousness of the land. Thus, the accuracy of the model was established. (5) All the land units of the study area can be calculated using the land imperviousness estimation model. The spatially distributed patterns of land imperviousness are then obtained.

\subsubsection{Spatial pattern analysis on impervious surfaces}

Based on the surface imperviousness index, the imperviousness of the land surface of Beijing was extracted. The spatial pattern of the urban impervious surface was then assessed. The distribution patterns of the impervious surfaces corresponding to different stages in the development of Beijing might be revealed. Furthermore, the impacts of different remote sensing data on the impervious surface patterns could be evaluated. The key landscape indexes developed by landscape ecology were then applied to quantify the impervious surface distributed patterns.

\section{Results}

\subsection{TM-based surface imperviousness modelling}

Using PCA analysis, five multiple regression models were established. Table 2 lists the calculated regression coefficients of the different regression analyses.

Comparing the various models described above, we demonstrated that the fourth model (Eq. (6)) was the most accurate for measuring surface imperviousness. In the fourth model, the standard deviation indices of brightness from bands 1-6 were considered. The basic spectral information from bands 1,5 , and 6 , and the standard deviation of band 6 contributed significantly to the measurements of the surface imperviousness (Eq. (6)).

$$
\begin{gathered}
Y=-3.087+1.803 \times \ln (b 1)-1.997 \times \ln (b 5)+ \\
1.003 \times \ln (b 6)-0.0009 \times \operatorname{std} 6
\end{gathered}
$$

where, $R=0.851, R^{2}=0.724$, and the adjusted $R^{2}=$ 0.720 . Using variance analysis $(F=203.073, P=0.0001$; $\alpha=0.05)$. We confirmed that this regression model was effective and reliable, as $72 \%$ of the surface imperiousness variance could be explained by the three variables.

Table 2 Parameters of multi-linear regression analysis on impervious index based on TM multi-spectral features

\begin{tabular}{llll}
\hline Index & $\begin{array}{l}\text { Multiple regression } \\
\text { correlation }(R)\end{array}$ & $R^{2}$ & $\begin{array}{l}\text { Adjusted } \\
R^{2}\end{array}$ \\
\hline NDVI, NDBI & 0.712 & 0.507 & 0.504 \\
$\ln (b 2), \ln (b 5), \ln (b 6)$ & 0.847 & 0.718 & 0.714 \\
$\ln (b 1), \ln (b 5), \ln (b 6)$, Std6 & 0.851 & 0.724 & 0.720 \\
Ratio4, Ratio5 & 0.829 & 0.687 & 0.683 \\
\hline
\end{tabular}


We chose to verify and validate the fourth regression model. An additional 110 sample units, totalling $31 \mathrm{~km}^{2}$ in area, were selected as reference samples, and the actual imperviousness was measured by interpreting the Quickbird high-resolution images. The accuracy assessments indicated that the relativity coefficient of the simulated imperviousness based on TM image and true imperviousness was 0.91 and the standard residual error was $5.64 \%$ (Fig. 2a, b).

In general, the overall surface imperviousness was somewhat lower than the real surface imperviousness. These results suggested that surface imperviousness estimated from the model in the less-developed areas coincided better with the real surface imperviousness but was underestimated in the well-developed areas. In the well-developed areas, similar spectral responses from some bright impervious surfaces and dry soils created situations where measurements were difficult to make with high confidence. Additionally, tree crowns that cover portions of some impervious surface, such as roads, may also have caused some underestimation of the impervious surfaces.

\subsection{SPOT-based surface imperviousness modelling}

For the SPOT image data, a total of 15 indices were developed to explore surface imperviousness. Using the PCR analysis, three multiple regression models were established (Tables 3 and 4). Among the three models, it could be seen
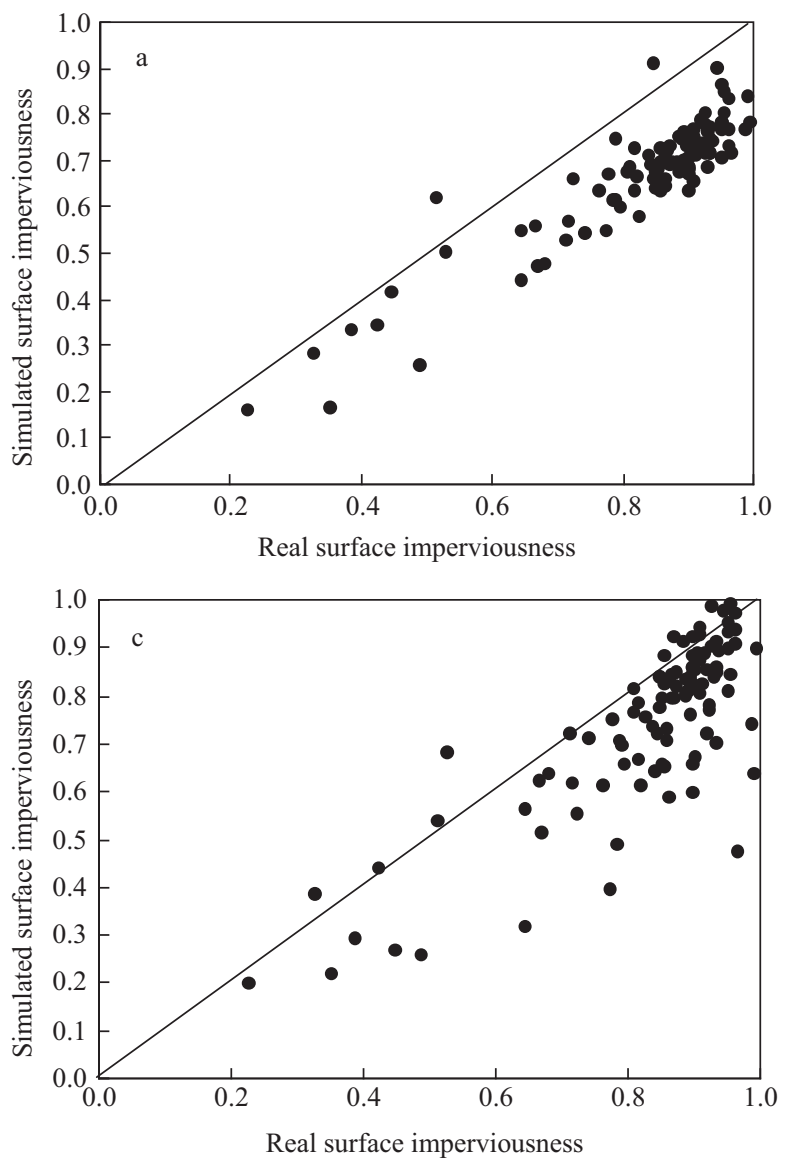

that the combination of NDVI, the near-infrared band, the standard deviation of the visible green band and the ratio of the short-wave infrared band could estimate the surface imperviousness with the highest multiple regression correlation (Eq. (7)):

$$
\begin{aligned}
Y= & 4.829-2.27 \mathrm{NDVI}-0.666 \ln (b 3)- \\
& 0.0046 \mathrm{std} 1-5.452 \text { Ratio4 }
\end{aligned}
$$

where, $R=0.818, R^{2}=0.670$, and the adjusted $R^{2}=0.663$. Using variance analysis $(F=95.277, P=0.0001 ; \alpha=$ $0.05)$. We confirmed that this regression model was effective and reliable, as $68.3 \%$ of the surface imperviousness variance could be explained by the three variables.

The validation result showed that the relativity coefficient of the simulated imperviousness based on SPOT image and real imperviousness was 0.81 and the standard residual error was $10.56 \%$ (Fig. 2c, d).

In general, the overall surface imperviousness coincided better with the real surface imperviousness but there was more scatter than that for the TM-based surface imperviousness. Analysis showed that the shadows cast by tall buildings lowered the value of the surface imperviousness estimated from the remote sensing data, while roofs made from materials such as asbestos could increase the estimated value of the surface imperviousness.
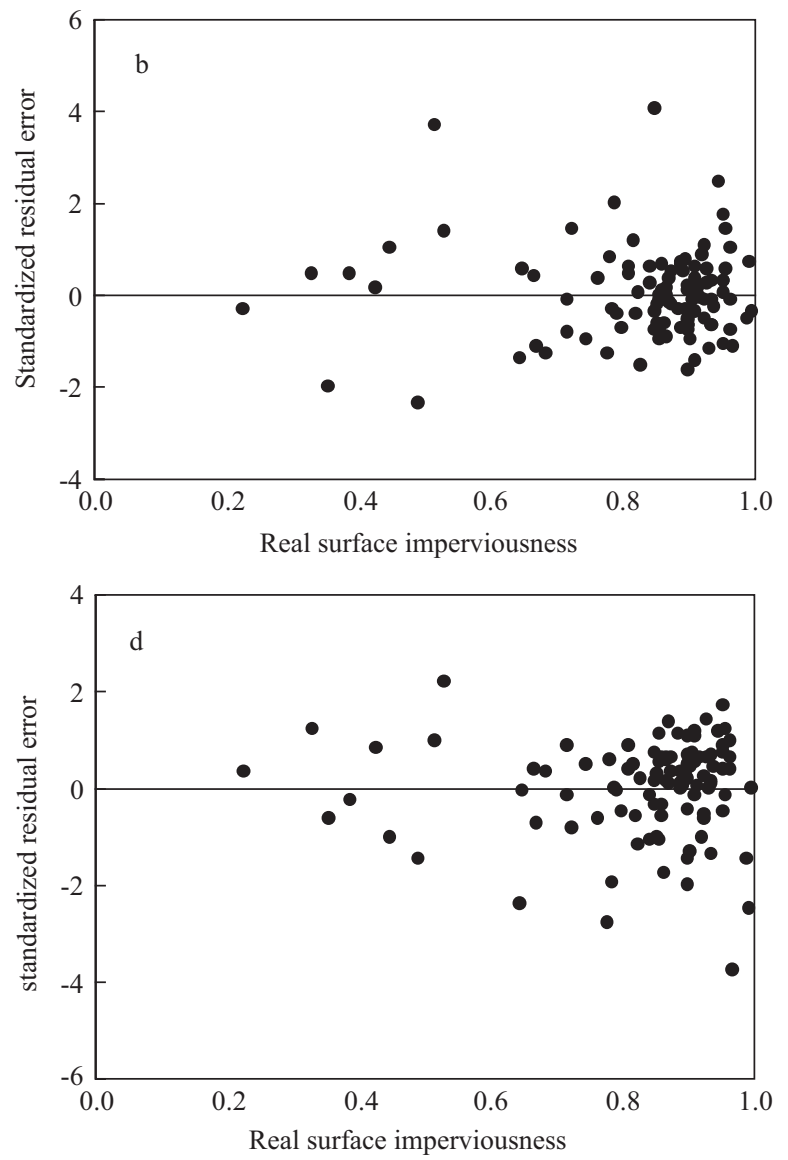

Fig. 2 Comparison of TM-based surface imperviousness with true imperviousness (a), accuracy assessment on TM-based surface imperviousness (b), comparison of SPOT-based surface imperviousness with true imperviousness (c), and accuracy assessment on SPOT-based surface imperviousness (d). 
Table 3 Relationship between impervious index and SPOT multi-spectral factors

\begin{tabular}{|c|c|c|c|c|c|c|c|c|}
\hline & $\ln (b 1)$ & $\ln (b 2)$ & $\ln (b 3)$ & $\ln (b 4)$ & $\ln \left(b_{r}\right)$ & Maxdiff & Ratio1 & Ratio2 \\
\hline $\begin{array}{l}\operatorname{Imp} \\
\ln (b 1) \\
\ln (b 2) \\
\ln (b 3) \\
\ln (b 4) \\
\ln \left(b_{r}\right) \\
\text { Max diff } \\
\text { Ratio1 }\end{array}$ & $0.452 * *$ & $\begin{array}{l}0.450 * * \\
0.983 * *\end{array}$ & $\begin{array}{l}-0.425^{* *} \\
0.318^{* *} \\
0.349^{* *}\end{array}$ & $\begin{array}{l}-0.077 \\
0.693 * * \\
0.736^{* *} \\
0.854 * *\end{array}$ & $\begin{array}{l}0.058 \\
0.829 * * \\
0.854 * * \\
0.779 * * \\
0.969 * *\end{array}$ & $\begin{array}{l}0.150 \\
-0.469 * * \\
-0.543 * * \\
-0.771 * * \\
-0.867 * * \\
-0.784 * *\end{array}$ & $\begin{array}{l}0.521^{* *} \\
-0.093 \\
-0.150 \\
-0.931^{* *} \\
-0.747^{* *} \\
-0.616^{* *} \\
0.735^{* *}\end{array}$ & $\begin{array}{l}0.730 * * \\
0.519 * * \\
0.508^{* *} \\
-0.584 * * \\
-0.157 \\
0.013 \\
0.246 * * \\
0.695 * *\end{array}$ \\
\hline & Ratio3 & Ratio4 & std 1 & $\operatorname{std} 2$ & std3 & std4 & NDVI & \\
\hline $\operatorname{Imp}$ & $-0.740 * *$ & $-0.327 * *$ & -0.144 & $-0.184 *$ & $-0.237 * *$ & -0.099 & $-0.763 * *$ & \\
\hline $\ln (b 1)$ & $-0.489 * *$ & $0.314 * *$ & $0.277 * *$ & $0.262 * *$ & 0.069 & $0.210^{*}$ & $-0.499 * *$ & \\
\hline $\ln (b 2)$ & $-0.467 * *$ & $0.381 * *$ & $0.242 * *$ & $0.248 * *$ & 0.036 & $0.193^{*}$ & $-0.483^{* *}$ & \\
\hline $\ln (b 3)$ & $0.641 * *$ & $0.794 * *$ & 0.091 & 0.147 & 0.117 & -0.025 & $0.651 * *$ & \\
\hline $\ln (b 4)$ & $0.189 *$ & $0.841 * *$ & 0.151 & $0.195^{*}$ & 0.038 & 0.115 & $0.200^{*}$ & \\
\hline $\ln \left(b_{r}\right)$ & 0.036 & $0.713 * *$ & $0.204 *$ & $0.234 * *$ & 0.076 & 0.126 & 0.036 & \\
\hline Max diff & $-0.255^{* *}$ & $-0.855^{* *}$ & -0.139 & $-0.202^{*}$ & -0.041 & -0.123 & $-0.281 * *$ & \\
\hline Ratio1 & $-0.736^{* *}$ & $-0.815^{* *}$ & -0.002 & -0.064 & -0.039 & 0.065 & $-0.749 * *$ & \\
\hline Ratio2 & $-0.936^{* *}$ & $-0.425^{* *}$ & 0.136 & 0.104 & -0.022 & $0.212^{*}$ & $-0.958 * *$ & \\
\hline Ratio3 & & $0.408 * *$ & -0.108 & -0.054 & 0.074 & $-0.199 *$ & $0.979 * *$ & \\
\hline Ratio4 & & & 0.059 & 0.108 & -0.032 & 0.064 & $0.432 * *$ & \\
\hline std1 & & & & $0.971 * *$ & $0.705 * *$ & $0.750 * *$ & -0.110 & \\
\hline $\operatorname{std} 2$ & & & & & $0.750 * *$ & $0.807 * * *$ & -0.062 & \\
\hline std3 & & & & & & $0.768 * *$ & 0.079 & \\
\hline std4 & & & & & & & $-0.180 *$ & \\
\hline
\end{tabular}

Imp: percentage of impervious surface; $\ln (b 1)-\ln (b 4): 1-4$ spectral channels of SPOT image by standardization; $\ln \left(b_{r}\right)$ : the channel mean value of 1-4 spectral bands by standardization; Ratio1-Ratio4: ratio of brightness of 1-4 spectral channels of SPOT image; std1-std4: standard derivation of brightness of channels 1-4 of SPOT image; Max diff: maximum difference of brightness.

Table 4 Parameters of multi-linear regression analysis on impervious index and SPOT multi-spectral features

\begin{tabular}{llll}
\hline Index & $\begin{array}{l}\text { Multiple regression } \\
\text { correlation }(R)\end{array}$ & $R^{2}$ & Adjust $R^{2}$ \\
\hline NDVI & 0.763 & 0.582 & 0.579 \\
$\ln (b 2), \ln (b 3)$ & 0.768 & 0.589 & 0.583 \\
NDVI, $\ln (b 3)$, std1, Ratio4 & 0.818 & 0.670 & 0.663 \\
\hline
\end{tabular}

\subsection{Spatial patterns of Beijing impervious surfaces}

The spatial patterns of urban surface imperviousness within inner city of Beijing were mapped by applying the imperviousness model derived from the fourth multiple regression model. The surface imperviousness estimates were further classified: (1) very low imperviousness (below 0.3); (2) low imperviousness (within the range $0.3-0.5$ ); (3) middle imperviousness (within the range 0.50.7 ); and (4) high imperviousness (above 0.7). Using these distinctions, the urban surface imperviousness was then mapped (Fig. 3a, b).

To quantitatively measure the surface impervious patterns, the representative landscape indices were selected, including percentage of landscape (PLAND) and the large shape index (LSI).

Over the entire spatial scale, the spatial patterns of impervious land surface based on both TM and SPOT images were somewhat similar. The imperviousness of the inner city was high, particularly within the fourth ring road, where the percentage of middle impervious patches and high impervious patches exceeded $70 \%$. This implied that the degree of urbanization of the inner city was very high, while the green space and water-body content were low. Furthermore, the surface imperviousness patterns varied considerably within the different ring belts. The heterogeneity of all four types of impervious patches increased from the inner centre to the rural edge. This suggested that, in recent decades, the spatial patterns of new developed land cover/use were diverse. For residential land use, the new residential properties developed in recent decades were relatively high (15-30 floors), however, they contributed less density. The residential properties developed in the 1980s were generally in the range 10-15 floors, with high density. Many older residential buildings developed several decades ago, were only one-story tall, with very high density. Furthermore, the spatial surface impervious patterns or non-residential land cover/use also differed significantly for different ring belts. Additional government buildings, institutions, universities, and large commercial buildings constructed in a centralized fashion between the third and fourth ring roads served to significantly increase the surface imperviousness. The edge of the inner city (between the fourth and fifth ring roads), represents the urban-rural transitional zone where the villages and modern industry have developed and mainly contributed to the impervious surface. Thus, the spatial distributed pattern of urban surface imperviousness is very helpful to recognize the city's development mode which is crucial for the environment.

Moreover, from the sub-spatial scale, the spatial patterns of impervious land surface based on both TM and SPOT images were obviously different (Fig. 4). The spatial patterns varied from the second to the fifth ring belt, for the very low, low, middle and high impervious surface classes. 


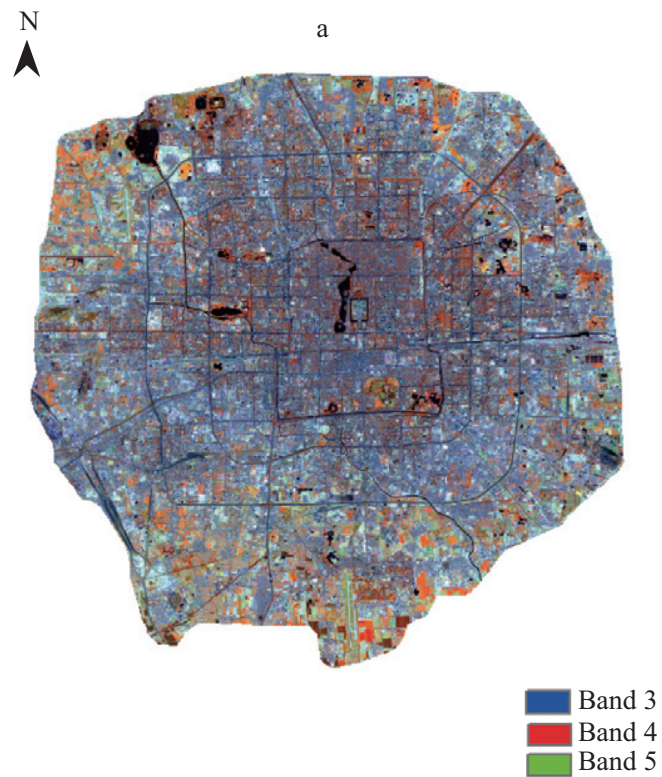

b

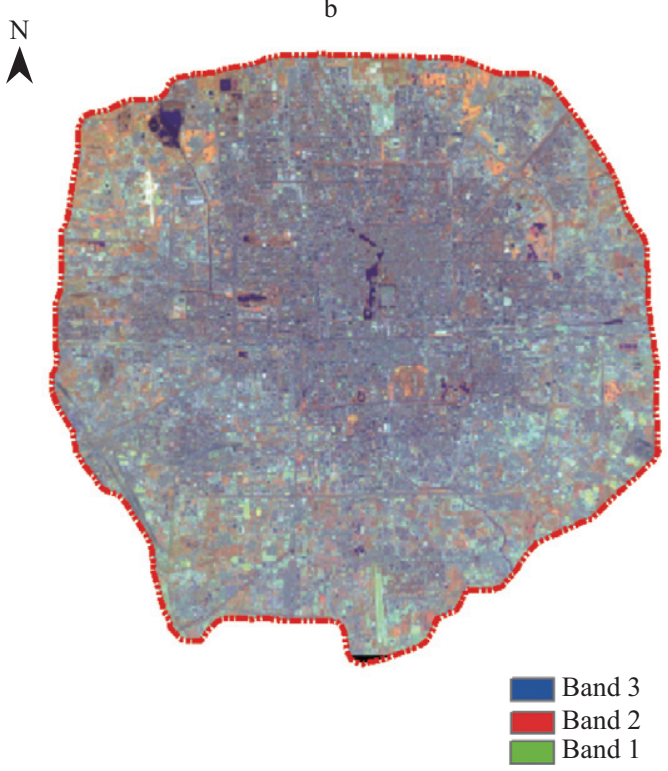

$\stackrel{\mathrm{N}}{\Lambda}$

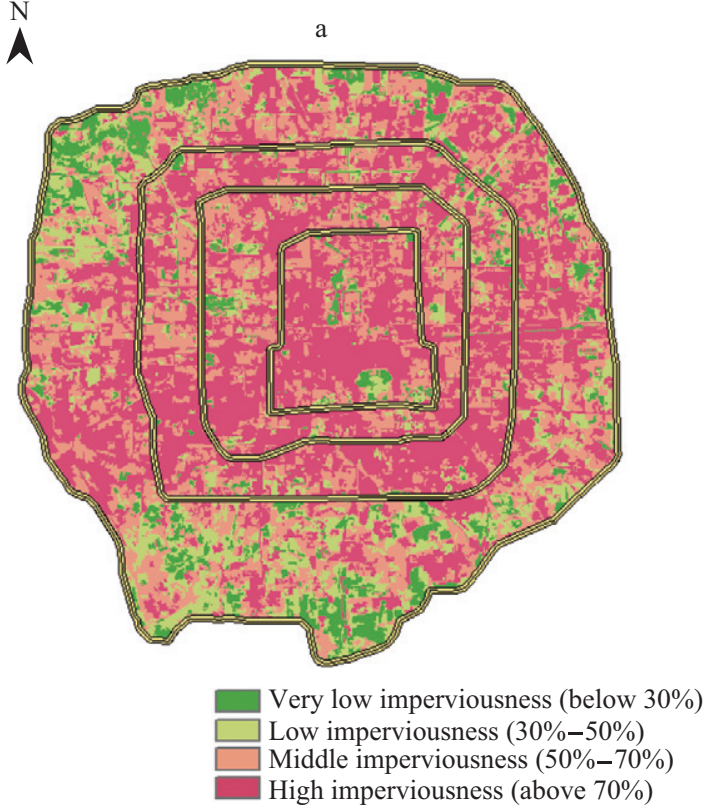

N

b

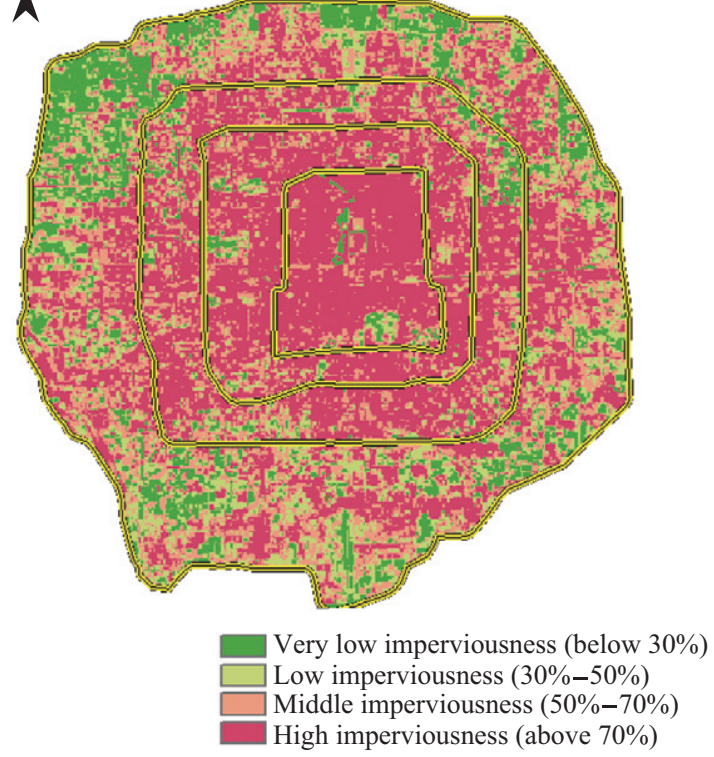

Fig. 3 Surface imperviousness pattern based on TM image (a) and surface imperviousness pattern based on SPOT image (b).

Within the second and third ring belts, the percentage of high impervious lands derived from the SPOT image was higher than that from the TM image. Similarly, within the fourth and fifth ring belts, the percentage of very low impervious lands derived from the SPOT image was also higher than that from the TM image. In contrast, from the second to fifth ring belts the percentage of middle surface impervious lands from SPOT image was lower than that from TM image. The estimates from the TM and SPOT images were similar for the low impervious lands.

For the landscape shape index, within the second ring belt, all four types of impervious land from the SPOT image were similar to those from the TM image. From the third to the fifth ring belts, all types of impervious lands from the SPOT image were higher than those from the TM image. Moreover, the further from the central core, the more the rate of the LSI increased.

\section{Discussion}

The surface imperviousness is a critical indicator of environment quality in urban settings. Methods are needed to accurately quantify the percentage of impervious surfaces in such areas. In this study, we used different remote sensing data and incorporated multi-spectral indicators to estimate the impervious surface fractions in a dense urban environment. This approach provides an effective way to accurately estimate the percentage of impervious surfaces in urban settings.

In contrast to traditional SMA that assumes the spectral responses from different types of impervious surfaces are constant (Philip and Roberts, 2003), this approach accounted for the variations of spectral responses of different impervious surfaces, and thus improved the estimations. The PCA and PCR analyses proved to be effective in pre- 

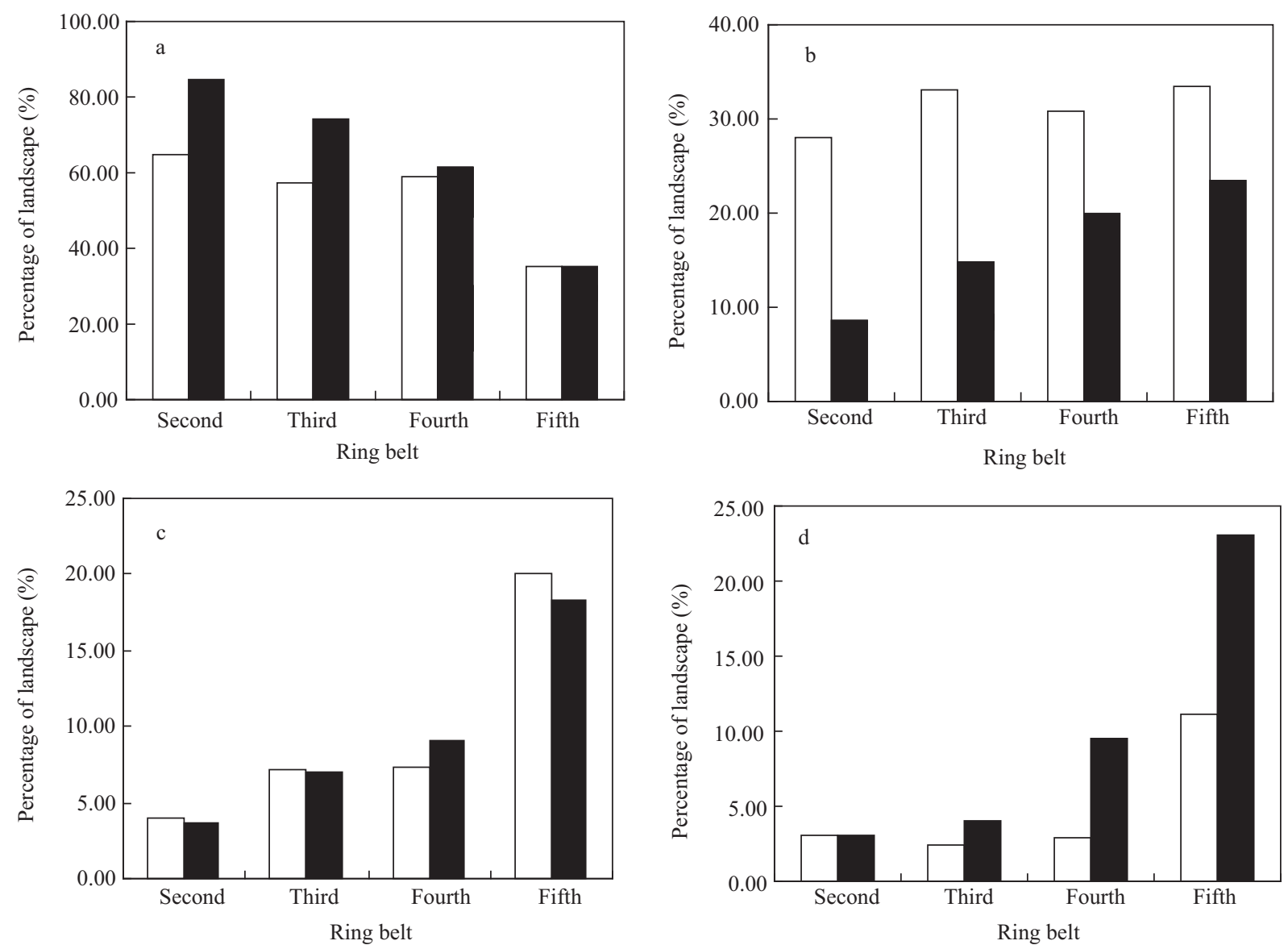

Fig. 4 PLAND of high (a), middle (b), low (c), and very low (d) impervious landscape patterns.

dictor selection and modelling. Furthermore, rather than using pixels as the unit of analysis, an object-based image analysis approach not only uses the spectral properties, but also the shape, texture, and context information. Compared to the pixel-based classification method, this approach might greatly reduce the variation within a specific class by averaging the individual pixel variation, and thus improve the estimate of the impervious surfaces (Zhou and Troy, 2008a, 2008b).

Our results highlight the need to combine data with different spatial resolution when quantifying the percentage of imperviousness, as higher resolution data do not necessarily lead to more accurate estimates. At the whole city level, the TM data better predict the percentage cover of impervious surfaces. However, the analyses at a finer scale (i.e., sub-city level) reveal that, depending on the degree of imperviousness, models using SPOT data provided better estimates than those using TM data for some, but not all, areas. The results clearly showed that the spatial patterns of the four types of impervious surfaces by ring belts derived from TM data differed greatly from those obtained from SPOT data. For example, within the third ring belt, the percentage of highly imperviousness land derived from SPOT was obviously higher than that from TM. This may be due to the higher built-up density within the third ring belt. The increased shadow effect on the SPOT image may lead to the surface imperviousness being overestimated. In contrast, the value for the percentage of middle imperviousness land within the third ring belt derived from SPOT was lower than that from TM. This is because there were more mixed pixels of pervious and impervious lands in the lower resolution TM data. Land with low imperviousness was mostly found at the fourth ring belts. The percentage of low imperviousness derived from SPOT data was similar to that from TM data, suggesting that both types of data work well for areas with low imperviousness. Areas with very low imperviousness, primarily urban green spaces, were distributed across the whole city, from the second to the fifth ring belt. The percentage of this land type derived from SPOT is higher than that from TM. The closer to the suburban ring belt, the larger the difference. The landscape in suburban areas was less fragmented, and there were more large patches of vegetation. In general, impervious surfaces within those patches of vegetation were not identified by the TM data. Consequently, the percentage of impervious surfaces was underestimated.

The results also indicated that the spatial resolution of image data has effects on spatial pattern analysis of impervious surfaces. For example, the LSI derived from SPOT was higher than that from TM for four types of imperviousness. Increasing spatial resolution can lead to an increase in the LSI. The effects of spatial resolution on landscape indices, however, decrease with the increase 
in the degree of landscape fragmentation. The more fragmented the landscape pattern, the less the effects of spatial resolution, and thus the less difference in landscape indices.

\section{Conclusions}

This study presents PCR models that use multispectral indicators to estimate the imperviousness of urban surfaces. These results highlight the need for multiple resolution data to quantify the percentage of imperviousness, as higher resolution data do not necessarily lead to more accurate estimates. Percentage of imperviousness should be accurately quantified by selecting remotely sensed data with appropriate resolution based on the specific research question. Our results also indicated that the spatial pattern of the impervious surfaces was closely related to the city's development. The distribution of impervious surfaces from the inner city to the suburbs revealed the characteristics of the development of the city, which was closely related to the regional eco-environmental changes. There results provide important insights for urban planning and land use decision-making.

\section{Acknowledgment}

This work was supported by the National Basic Research Program (973) of China (No. 2008CB418104), the Major Programs of the Chinese Academy of Sciences (No. KZCX1-YW-14-4-1), and the National Natural Science Foundation of China (No. 40901265).

\section{References}

Abdul-Wahab S A, Bakheit C S, Al-Alawi S M, 2005. Principal Component and multiple regression analysis in modeling of ground-level ozone and factors affecting its concentrations. Environmental Modelling $\mathcal{G}$ Software, 20: 1263-1271.

Bauer M E, Heinert N J, Doyle J K, Yuan F, 2004. Impervious surface mapping and change monitoring using satellite remote sensing. In: Proceedings of ASPRS Annual Conference. Denver, Colorado. 24-28 May.

Civco J D, Hurd E H, Wilson C L, Arnold M P, Prisloe J, 2002. Quantifying and describing urbanizing landscapes in the northeast United States. Photogrammetric Engineering and Remote Sensing, 68(10): 1083-1090.

Fernandez N, Paruelo J M, Delibes M, 2010. Ecosystem functioning of protected and altered Mediterranean environments: A remote sensing classification in Donana, Spain. Remote Sensing of Environment, 114: 211-220.

Flanagan M, Civco D L, 2001. Subpixel impervious surface mapping. In: Proceedings of ASPRS Annual Conference. St. Louis, MO. 23-27 April.

Goetz S J, Wright R K, Smith A J, Zinecker E, Schaub E, 2003. IKONOS imagery for resource management: Tree cover, impervious surfaces, and riparian buffer analyses in the mid-Atlantic region. Remote Sensing of Environment, 88: 195-208.

Lee J, Pak G, Yoo C, Kim S, Yoon J, 2010. Effects of land use change and water reuse options on urban water cycle. Journal of Environmental Sciences, 22(6): 923-928.
Lu D, Weng Q, 2006. Spectral mixture analysis of ASTER images for examining the relationship between urban thermal features and biophysical descriptors in Indianapolis, Indiana, USA. Remote Sensing of Environment, 104(2): 157-167.

Luo H B, Luo L, Huang G, Liu P, Li J, Hu S et al., 2009. Total pollution effect of urban surface runoff. Journal of Environmental Sciences, 21(9): 1186-1193.

Philip E D, Roberts D A, 2003. Endmember selection for multiple endmember spectral mixture analysis using endmember average RMSE. Remote Sensing of Environment, 87: 123135.

Ridd M K, 1995. Exploring a V-I-S (Vegetation-Impervious surface-soil) model for urban ecosystem analysis through remote sensing: Comparative anatomy for cities. International Journal of Remote Sensing, 16(12): 2165-2185.

Statheropoulos M, Vassiliadis N, Pappa A, 1998. Principal component and canonical correlation analysis for examining air pollution and meteorological data. Atmospheric Environment, 32: 1087-1095.

Vaidya O C, Howell G D, Leger D A, 2000. Evaluation of the distribution of mercury in lakes in Nova Scotia and Newfoundland. Water Air and Soil Pollution, 117: 353-369.

Wang Y Q, Zhang X, Lampa W, 2000. Improvement of spatial accuracy in natural resources mapping using multisensor remote sensing and multisource spatial data. In: Proceedings of the 4th International Symposium on Spatial Accuracy Assessment in natural resources and environmental sciences. Amsterdam, the Netherlands. 23-30 July.

Ward D, Phinn S R, Murry A T, 2000. Monitoring growth in rapidly urbanized areas using remotely sensed data. The Professional Geographer, 52(3): 371-386.

White M, Greer K A, 2006. The effects of watershed urbanization on the stream hydrology and riparian vegetation of Los Penasquitos Creek, California. Landscape and Urban Planning, 74(2): 125-138.

$\mathrm{Wu} \mathrm{C}, 2004$. Normalized spectral mixture analysis for monitoring urban composition using ETM+ imagery. Remote Sensing of Environment, 93: 480-492.

Xiao R B, Ouyang Z Y, Zheng H, Li W F, Erich S, Wang X $K$, 2007. Spatial pattern of impervious surface and their impacts on land surface temperature in Beijing, China. Journal of Environmental Sciences, 19(2): 250-256.

Yang L M, Huang C Q, Homer C G, Wylie B K, Coan M J, 2003. An approach for mapping large-area impervious surfaces: Synergistic use of Landsat 7 ETM+ and high spatial resolution imagery. Canadian Journal of Remote Sensing, 29(2): 230-240.

Yuan F, Bauer M E, 2007. Comparision of impervious surface area and normalized difference vegetation index as indicators of surface urban heat island effects in Landsat imagery. Remote Sensing of Environment, 106: 375-386.

Zha Y, Gao J, Ni S, 2003. Use of normalized difference builtup index in automatically mapping urban areas from TM imagery. International Journal of Remote Sensing, 24(3): 583-594.

Zhou W Q, Troy A, Grove M, 2008. Object-based land cover classification and change analysis in the Baltimore Metropolitan Area using multitemporal high resolution remote sensing data. Sensors, 8: 1613-1636.

Zhou W Q, Troy A, 2008. An object-oriented approach for analysing and characterizing urban landscape at the parcel level. International Journal of Remote Sensing, 29(11): 3119-3135. 